\title{
Prevalence of Spina Bifida Occulta and Its Relationship With Overactive Bladder in Middle-Aged and Elderly Chinese People
}

\author{
Jun Wei Wu ${ }^{1,2, *}$, Yu Rong Xing,**, Yi Bo Wen ${ }^{1}$, Tian Fang Li ${ }^{4}$, Jia Feng Xie ${ }^{1,2}$, Quan De Feng ${ }^{1,2}$, Xiao Ping Shang ${ }^{5}$, Yun Long Li1 ${ }^{1,2}$, \\ Jin Jin Feng ${ }^{1,2}$, Xin Xin Wang ${ }^{6}$, Rong Qun Zhai ${ }^{1,2}$, Xiang Fei He ${ }^{1,2}$, Tao Chen ${ }^{1,2}$, Xin Jian Liu ${ }^{1,2}$, Jian Guo Wen ${ }^{1,2}$ \\ ${ }^{1}$ Urodynamic Centre, Department of Urology, The First Affiliated Hospital of Zhengzhou University, Zhengzhou, China \\ ${ }^{2}$ Key-Disciplines Laboratory Clinical-Medicine Henan, Zhengzhou, China \\ ${ }^{3}$ Center of Health Examination, The First Affiliated Hospital of Zhengzhou University, Zhengzhou, China \\ ${ }^{4}$ Department of Rheumatology \& Immunology, The First Affiliated Hospital of Zhengzhou University, Zhengzhou, China \\ ${ }^{5}$ Medical Record Department, The First Affiliated Hospital of Zhengzhou University, Zhengzhou, China \\ ${ }^{6}$ Reproductive Medical Centre, The First Affiliated Hospital of Zhengzhou University, Zhengzhou, China
}

Purpose: To investigate the prevalence of spina bifida occulta $(\mathrm{SBO})$ and its relationship with the presence of overactive bladder $(\mathrm{OAB})$ in middle-aged and elderly people in China.

Methods: A cross-sectional community-based survey was carried out at 7 communities in Zhengzhou City, China from December 15, 2013 to June 10,2014, where residents aged over 40 years were randomly selected to participate. All of the participants underwent lumbosacral radiographic analysis and relevant laboratory tests. A questionnaire including basic information, past medical history and present illness, and the $\mathrm{OAB}$ symptom score was filled out by all participants. Chi-square tests and logistic regression were used for data analysis with a $\mathrm{P}$-value of $<0.05$ denoting statistical significance.

Results: A total of 1,061 subjects were qualified for the final statistical analysis (58.8 \pm 11.7 years; male, 471 [44.4\%]; female, 590 [55.6\%]). The overall prevalence of SBO was 15.1\% (160 of 1,061): 18.3\% (86 of 471) in men and 12.5\% (74 of 590) in women. Among these subjects, $13.7 \%$ (145 of 1,061) had OAB: 13.2\% (62 of 471) in men and 14.1\% (83 of 590) in women. The results of logistic regression showed that age, $\mathrm{SBO}$, history of cerebral infarction (HCI), and constipation were risk factors for $\mathrm{OAB}(\mathrm{P}<0.05)$, while sex, history of childhood enuresis $(\mathrm{HCE})$, body mass index $(\mathrm{BMI})$, and diabetes mellitus $(\mathrm{DM})$ were not $(\mathrm{P}>0.05)$. In men, age, $\mathrm{SBO}$, and constipation were risk factors for $\mathrm{OAB}(\mathrm{P}<0.05)$, while $\mathrm{HCE}, \mathrm{BMI}, \mathrm{DM}, \mathrm{HCI}$, and benign prostate hyperplasia were not $(\mathrm{P}>0.05)$. In women, age, $\mathrm{SBO}$, and $\mathrm{HCI}$ were risk factors for $\mathrm{OAB}(\mathrm{P}<0.05)$, while $\mathrm{HCE}$, $\mathrm{BMI}, \mathrm{DM}$, vaginal delivery, and constipation were not $(\mathrm{P}>0.05)$.

Conclusions: The prevalence of SBO is high and it is related to $\mathrm{OAB}$ in middle-aged and elderly people in China.

Keywords: Prevalence; Spina Bifida Occulta; Urinary Bladder, Overactive; Aged

- Fund/Grant Support: This study was supported by the National Science Foundation of China (81373869) and the Scientific Research Foundation for the Doctoral Program of Zhengzhou University.

- Research Ethics: This research has been approved by the Medical Research Ethics Committee of The First Affiliated Hospital of Zhengzhou

University, and the authorization number is Scientific Research 49th, 2013.

- Conflict of Interest: No potential conflict of interest relevant to this article was reported.

Corresponding author: Jian Guo Wen (iD http://orcid.org/0000-0003-0952-118X Urodynamic Centre, Department of Urology, the First Affiliated Hospital of Zhengzhou University; Key-Disciplines Laboratory Clinical-Medicine Henan, Zhengzhou 450052, Henan, China

E-mail: wenjg@hotmail.com / Tel: +86-371-66295228 / Fax: +86-371-66970906

*Jun Wei Wu and Yu Rong Xing contributed equally to this study as co-first authors.

Submitted: October 21, 2015 / Accepted after revision: February 22, 2016 


\section{INTRODUCTION}

Spina bifida occulta (SBO) is a subtle form of dysraphism with one or more split spinous processes and widened interpedicular distances on plain radiography [1]. These major pathologies combined with other abnormalities, though relatively uncommon, form the so-called tethered cord syndrome (TCS). These abnormalities include cutaneous changes (midline lumbosacral cutaneous hemangiomas, lumbosacral hypertrichosis, lumbosacral dermal sinus, and skin appendages), neurological and orthopedic changes (sensory loss and structural deformities of the feet, limb-length abnormalities, muscular atrophy of the legs, gait disturbance, limb pain, and scoliosis), bladder/bowel dysfunction (repeated urinary tract infection [UTI], voiding dysfunction, bowel incontinence), vertebral anomalies (bifid vertebrae, laminar defects, hemivertebrae), and anorectal and urogenital malformations (imperforate anus, renal dysplasia, bladder exstrophy) [1-3].

The majority of subjects with SBO of the lower spine and sacrum do not have clinical manifestations such as neurological abnormalities and cutaneous/subcutaneous malformations. However, Galloway and Tainsh [4] demonstrated an increased prevalence of SBO in a small group of adults with lower urinary tract problems. Furthermore, Fidas et al. [5] revealed a significant increase in the prevalence of SBO at the levels of S1 and S2 in patients with urological symptoms, including urgency and instability in men and stress incontinence and urgency in women. However, both studies had, to some extent, design issues. One did not perform statistical analysis and the other did not exclude confounding factors such as benign prostate hyperplasia (BPH), diabetes, and UTIs. Clearly, more comprehensive studies are warranted to delineate the relationship between $\mathrm{SBO}$ and lower urinary tract symptoms (LUTS). Using multiple methodologies, our current study aimed to establish the prevalence of $\mathrm{SBO}$ and its relationship with $\mathrm{OAB}$ through screening of middle-aged and elderly Chinese people.

\section{MATERIALS AND METHODS}

We randomly selected 7 communities in Zhengzhou City from December 15, 2013 to June 10, 2014 and assigned a serial number to every member of the community over 40 years old. We then used a random number table to randomly choose 170 persons in each community. Finally, 1,190 samples were selected and 1,061 people participated in this survey after applying the exclusion criteria. The exclusion criteria included pregnancy; seniors lacking communication capability due to dementia; paraplegics; a history of spinal disease; past surgeries on the prostate, urethra, or bladder; obscure digital radiography because of intestinal gas; a history of diuretics therapy; inconsistent diagnoses between the 2 radiologists.

All of the participants underwent lumbosacral radiography, routine urine tests, an oral glucose tolerance test (OGTT), and ultrasonography of the urinary system to establish the diagnosis of SBO and to identify cases of UTI, diabetes mellitus (DM), and $\mathrm{BPH}$ to exclude any potential confounding factors. A questionnaire including basic information such as past medical history, present illness, and the OAB symptom score (OABSS) was filled out by each participant.

The diagnostic criteria of $\mathrm{OAB}$ were established as an urgency score of OABSS $\geq 2$ and a total score $\geq 3$ [6]. DM was defined as follows: (1) Previously diagnosed with DM; (2) If the fasting blood glucose was above $7.0 \mathrm{mmol} / \mathrm{L}$, an OGTT was administered and 2-hour postprandial blood glucose $>11.1 \mathrm{mmol} / \mathrm{L}$ was diagnosed as diabetes. The prostate volume was estimated as transverse diameter $\times$ anteroposterior diameter $\times$ length $\times 0.52$, and $\mathrm{BPH}$ was diagnosed if the prostate volume was $>25 \mathrm{~cm}^{3}$.

Plain spinal and sacral radiographs obtained from all subjects were assessed by 2 senior radiologists for the presence or absence of fusion of the posterior elements of the lumbar and/ or sacral vertebrae above S3 [7]. No evident abnormalities were found during physical examinations.

IBM SPSS Statistics ver. 21.0 (IBM Co., Armonk, NY, USA) was used for data analysis. Chi-square tests were used to determine the differences of prevalence between sex, age groups, and people with $\mathrm{SBO}$ or $\mathrm{OAB}$. A stepwise multivariable logistic regression analysis was used to control for potential confounding variables and to determine risk factors associated with $\mathrm{OAB}$. A P-value of $<0.05$ was denoted as statistically significant.

\section{RESULTS}

Altogether 1,061 subjects were qualified for the final statistical analysis ( $58.8 \pm 11.7$ years; 471 men and 590 women). The overall prevalence of SBO in men was $18.3 \%$ (86 of 471) and in women, $12.5 \%$ (74 of 590). The prevalence of SBO was significantly higher in men than in women $(\mathrm{P}<0.05)$. To determine the role of aging in the formation of $\mathrm{SBO}$, the patients were divided into the following groups: 40-49, 50-59, 60-69, 70-79, and $\geq 80$ years. The SBO prevalence of total cases and by sex in 
each age group is shown in Table 1. No significant difference of SBO prevalence was found among the different age groups $(\mathrm{P}>$ $0.05)$ in either sex. S1 was identified as the most frequent location of SBO, accounting for 79.4\% (127 of 160) of cases, while the prevalences of SBO at S2, L5-S1, S1-S2, and L5 were 6.8\% (11 of 160), $6.3 \%$ (10 of 160), 4.4\% (7 of 160), and 3.1\% (5 of 160), respectively.

A total of 145 cases with $\mathrm{OAB}$ were identified, an overall prevalence of $13.7 \%$. No significant difference in the prevalence was found between men $(13.2 \%, 62$ of 471$)$ and women (14.1\%, 83 of 590), $\mathrm{P}>0.05$. Of note, the prevalence of $\mathrm{OAB}$ increased

Table 1. The incidence of SBO in total cases, male and females by decades

\begin{tabular}{llll}
\hline Age $(\mathrm{yr})$ & Total $(\%)$ & Male $(\%)$ & Female (\%) \\
\hline $40-49$ & $18.9(43 / 227)$ & $22.7(30 / 132)$ & $13.7(13 / 95)$ \\
$50-59$ & $13.4(50 / 374)$ & $14.2(21 / 148)$ & $12.8(29 / 226)$ \\
$60-69$ & $12.7(29 / 229)$ & $18.1(15 / 83)$ & $9.6(14 / 146)$ \\
$70-79$ & $15.9(30 / 189)$ & $18.1(15 / 83)$ & $14.2(15 / 106)$ \\
$\geq 80$ & $19.0(8 / 42)$ & $20.0(5 / 25)$ & $17.7(3 / 17)$ \\
\hline
\end{tabular}

SBO, spina bifida occulta. with age: $6.6 \%$ (15 of 227), 9.1\% (34 of 374), 14.8\% (34 of 229), $24.3 \%$ ( 46 of 189 ), and $38.1 \%$ ( 16 of 42 ) for the groups aged 40 $49,50-59,60-69,70-79$, and $\geq 80$ years old, respectively. The prevalence of $\mathrm{OAB}$ was significantly lower in the 50-59 age group subjects than those in the 60-69 and 70-79 groups $(\mathrm{P}<0.05)$, while no difference was detected among the other age groups. Altogether $54 \mathrm{OAB}$ cases also had $\mathrm{SBO}$ and the prevalence of $\mathrm{OAB}$ in the subjects with $\mathrm{SBO}$ was $33.8 \%$ (54 of $160)$, which was significantly higher than for those without SBO $(10.1 \%$, 91 of $901 ; \mathrm{P}<0.0001)$. The prevalence of SBO in the subjects with $\mathrm{OAB}$ was $37.2 \%$ (54 of 145), which was significantly higher than in those without $\mathrm{OAB}(11.6 \%, 106$ of 916; $\mathrm{P}<0.0001$ ).

When we investigated the relationship between different locations of $\mathrm{SBO}$ and $\mathrm{OAB}$ severity, we found no significant difference of $O A B$ prevalence among the different locations of $\mathrm{SBO}(\mathrm{P}>0.05)$. However, there were also no significant differences among groups $\mathrm{S} 2$ and $\mathrm{L} 5$ and the no-SBO group $(\mathrm{P}>$ $0.05)$, which may be due to the limited number of persons in groups L5 and S2 (Table 2).

Among the 1,061 subjects, there were 40 with a history of history of cerebral infarction (HCI), 91 with history of childhood

Table 2. The information of the relationship among different location of $\mathrm{SBO}$ and the $\mathrm{OAB}$ severity

\begin{tabular}{lcccc}
\hline Location of SBO & No. of SBO & No. of OAB & OAB in each group (\%) & Moderate or severe OAB (OABSS $\geq 6), \mathrm{n}(\%)$ \\
\hline S1 & 127 & 44 & 34.6 & $28(22.0)$ \\
S2 & 11 & 2 & 18.2 & $2(18.2)$ \\
L5 & 5 & 2 & 40.0 & $1(20.0)$ \\
L5+S1/S1+S2 & 17 & 6 & 35.3 & $5(29.4)$ \\
No. of SBO & - & 91 & $10.1(91 / 901)$ & $72(8.0)$ \\
\hline
\end{tabular}

SBO, spina bifida occulta; $\mathrm{OAB}$, overactive bladder; OABSS, OAB symptom score.

Table 3. The results of logistic regression in total samples

\begin{tabular}{lcccccc}
\hline Factor & $\beta$ & SE & Wald & P-value & OR & $95 \%$ CI \\
\hline Age & 0.05364 & 0.00831 & 6.456 & $<0.001$ & 1.06 & $1.04-1.07$ \\
Sex & 0.18610 & 0.19845 & 0.938 & 0.348 & 1.20 & $0.82-1.78$ \\
SBO & 1.59191 & 0.21806 & 7.300 & $<0.001$ & 4.91 & $3.2-7.53$ \\
HCE & 0.29635 & 0.32645 & 0.908 & 0.364 & 1.34 & $0.71-2.55$ \\
BMI & 0.07847 & 0.27871 & 0.282 & 0.778 & 1.08 & $0.63-1.87$ \\
DM & -0.58676 & 0.33779 & -1.737 & 0.082 & 0.56 & $0.29-1.08$ \\
HCI & 0.98403 & 0.37892 & 2.597 & 0.009 & 2.68 & $1.27-5.62$ \\
Constipation & 0.70356 & 0.70356 & 2.460 & 0.014 & 2.02 & $1.15-3.54$ \\
\hline
\end{tabular}

SE, standard error; OR, odds ratio; CI, confidence interval; SBO, spina bifida occulta; HCE, history of childhood enuresis; BMI, body mass index; $\mathrm{DM}$, diabetes mellitus; HCI, history of cerebral infarction. 
Table 4. The results of logistic regression in males

\begin{tabular}{|c|c|c|c|c|c|c|}
\hline Factor & $\beta$ & SE & Wald & P-value & OR & $95 \% \mathrm{CI}$ \\
\hline Age & 0.05022 & 0.01304 & 3.850 & $<0.001$ & 1.05 & $1.02-1.08$ \\
\hline $\mathrm{SBO}$ & 1.33603 & 0.33243 & 4.019 & $<0.001$ & 3.80 & $1.98-7.30$ \\
\hline HCE & 0.21904 & 0.39718 & 0.551 & 0.581 & 1.24 & $0.57-2.71$ \\
\hline BMI & 0.04912 & 0.51523 & 0.168 & 0.619 & 0.70 & $0.51-1.47$ \\
\hline $\mathrm{DM}$ & -0.98762 & 0.53614 & -1.842 & 0.065 & 0.37 & $0.13-1.07$ \\
\hline $\mathrm{HCI}$ & -0.19162 & 0.69159 & -0.277 & 0.782 & 0.83 & $0.21-3.20$ \\
\hline Constipation & 0.99504 & 0.45723 & 2.176 & 0.030 & 2.70 & $1.10-6.63$ \\
\hline $\mathrm{BPH}$ & 0.5711 & 0.36936 & 1.546 & 0.122 & 1.77 & $0.86-3.65$ \\
\hline
\end{tabular}

SE, standard error; OR, odds ratio; CI, confidence interval; SBO, spina bifida occulta; HCE, history of childhood enuresis; BMI, body mass index; $\mathrm{DM}$, diabetes mellitus; $\mathrm{HCI}$, history of cerebral infarction; $\mathrm{BPH}$, benign prostate hyperplasia.

Table 5. The results of logistic regression in females

\begin{tabular}{|c|c|c|c|c|c|c|}
\hline Factor & $\beta$ & SE & Wald & P-value & OR & $95 \%$ CI \\
\hline Age & 0.05007 & 0.01268 & 3.950 & $<0.001$ & 1.05 & $1.03-1.08$ \\
\hline $\mathrm{SBO}$ & 1.94203 & 0.29864 & 6.503 & $<0.001$ & 6.97 & $3.88-12.52$ \\
\hline $\mathrm{HCE}$ & 0.50058 & 0.56813 & 0.881 & 0.378 & 1.65 & $0.54-5.02$ \\
\hline BMI & 0.08467 & 0.48712 & 0.236 & 0.425 & 1.37 & $0.74-1.92$ \\
\hline $\mathrm{DM}$ & 0.40827 & 0.46052 & 0.887 & 0.375 & 0.66 & $0.27-1.64$ \\
\hline $\mathrm{HCI}$ & 1.68404 & 0.48866 & 3.446 & $<0.001$ & 5.39 & $2.07-14.04$ \\
\hline Constipation & 0.50077 & 0.38834 & 1.290 & 0.197 & 2.70 & $1.10-6.63$ \\
\hline VD & 0.07382 & 0.58035 & 0.127 & 0.899 & 1.65 & $0.77-3.53$ \\
\hline
\end{tabular}

SE, standard error; OR, odds ratio; CI, confidence interval; SBO, spina bifida occulta; HCE, history of childhood enuresis; BMI, body mass index; $\mathrm{DM}$, diabetes mellitus; HCI, history of cerebral infarction; VD, vaginal delivery.

enuresis (HCE), 95 with constipation, 99 with DM, 271 with BPH (81 with no symptoms), and 543 with vaginal delivery.

Logistic regression analysis demonstrated that in the general population, age, $\mathrm{SBO}, \mathrm{HCI}$, and constipation were risk factors for OAB $(\mathrm{P}<0.05)$, while sex, HCE, BMI, and DM were not $(\mathrm{P}>0.05)$ (Table 3). In men, age, SBO, and constipation were risk factors for $\mathrm{OAB}(\mathrm{P}<0.05)$, while $\mathrm{HCE}, \mathrm{BMI}, \mathrm{DM}, \mathrm{HCI}$, and $\mathrm{BPH}$ were not $(\mathrm{P}>0.05)$ (Table 4$)$. In women, age, $\mathrm{SBO}$, and HCI $(\mathrm{P}<0.05)$ were risk factors for OAB, but HCE, BMI, DM, constipation, and vaginal delivery were not $(\mathrm{P}>0.05)$ (Table 5).

Since previous studies have reported that $\mathrm{BPH}$ is one of the risk factors for $\mathrm{OAB}$, which is different from our conclusion, we also subdivided the cases with BPH using the severity score from the International Prostate Symptom Score (IPSS Form) to identify whether moderate or severe $\mathrm{BPH}$ was associated with $\mathrm{OAB}$. There were 271 men with BPH $(151,94$, and 26 men had an IPSS $\leq 7, \geq 8$ but $\leq 19$, and $\geq 20$ but $\leq 35$, respectively). The incidence of $\mathrm{OAB}$ in men with mild $\mathrm{BPH}$, moderate or severe
$\mathrm{BPH}$, and men without $\mathrm{BPH}$ was $5.3 \%$ (8 of 151 ), 35.8\% (43 of 120 ), and $5.5 \%$ (11 of 200), respectively. Although the prevalence of $\mathrm{BPH}$ was not related to $\mathrm{OAB}$, the incidence of $\mathrm{OAB}$ in moderate or severe $\mathrm{BPH}$ was significantly higher than in men with mild $\mathrm{BPH}$ or without $\mathrm{BPH}$.

\section{DISCUSSION}

SBO was first described and treated in 1891 by Jones, and surgery was suggested as a treatment by Brickner in 1918 [2]. Subsequently, increasingly more articles about the classification of and clinical manifestations of SBO were reported by neurosurgeons and urologists. SBO includes a large number of malformations including meningocele, meningocele manque, spinal lipomas, neurenteric cysts, split cord malformation, fatty filum terminale, terminal (lower one-third of the spinal cord) syrinx and dermal sinus tract, and simple nonfusion of the vertebral arches [8]. Generally, simple nonfusion of the vertebral arches 
accounts for the majority of SBO cases. Different subcategories display diverse clinical features. Because of the different characteristics and different locations of spinal cord lesions, SBO can affect multiple systems. However, it can also manifest as a single symptom such as nocturnal enuresis, repeated UTIs, or voiding dysfunction [9].

$\mathrm{SBO}$ is a relatively common anatomical variant that in one study was identified in $22 \%$ of the population (patients presenting to an emergency treatment service) ranging in age from 2 months to 98 years [10]. Other reports of its prevalence vary from $10 \%$ to $58 \%[7,11,12]$. These discrepancies may result from degenerative changes in seniors during the aging process. Differences between the races and sexes may also play a role.

Radiographic studies by Sutow and Pryde [12] for the first time demonstrated significant differences of the SBO prevalences among different age groups and that the prevalence decreased with age. Fidas et al. [7] also reviewed plain radiographs of the lumbosacral spine of 2,707 normal adult volunteers and they found that the prevalences of SBO in men, women, and all adults were $30 \%, 17 \%$, and $23 \%$, respectively. While the prevalence is almost double in men compared to women younger than 60 years, it becomes almost equal when the subjects are over 60 years of age [7]. The higher SBO prevalence in younger adults may be attributed to new bone formation or calcification as a result of degenerative processes [13]. A meta-analysis suggests that the most accurate estimate of the $\mathrm{SBO}$ incidence is $17 \%$, with the lumbosacral spine being the most frequently affected site. Laminar defects in the cervicothoracic spine are more common in patients of African descent compared to other races [14], suggesting race is a factor contributing to different prevalences of SBO.

We for the first time investigated the prevalence and the lesion sites of SBO among the Chinese people. Working with professional statisticians, we tried to design our study to make the results more reliable. We performed a cross-sectional study to evaluate the prevalence of SBO in normal adults. By doing so, we expected to exclude many confounding factors that arise in retrospective studies, such as spinal diseases [7].

Our cross-sectional, cross-sectional survey was carried out in a randomized, double-blind way in combination with a selfadministered questionnaire for the subjects aged over 40 years. All of the subjects were subjected to radiographic and other examinations in our hospital. Our results showed that the overall prevalence of SBO among the Chinese aged over 40 years was $15.1 \%$ (160 of 1,061), and its prevalence in men was $18.3 \%$ and
$12.5 \%$ in women. The prevalence of SBO was significantly higher among men than in women $(\mathrm{P}<0.05)$. Consistent with the meta-analysis mentioned above regarding the impact of race on SBO formation, one age-matched (over 40 years) study revealed a significantly higher prevalence of $\mathrm{SBO}$ in a Chinese population than in a British one $(15.1 \%$ vs. $9.8 \%, \mathrm{P}<0.05)$ [10].

The studies exploring the relationship between $\mathrm{SBO}$ and spinal cord abnormalities have generated inconsistent results, and the importance of radiographic SBO in patients with LUTS remains largely unknown. While some scientists suggest that children with SBO are more vulnerable to LUTS, others think that SBO may be a coincidental finding. Its clinical manifestations range from one to multiple symptoms. Reported bladder abnormalities in SBO include a detrusor hyperreflexia during filling in $42 \%$, low compliance detrusor in $67 \%$, and impaired bladder sensation in 8\%. Urethral abnormalities in SBO included detrusor/sphincter dyssynergia in $27 \%$, low pure max in $17 \%$, absent bulbocavernosus reflex in $56 \%$, and absent anal reflex in 57\% [9]. In contrast, Samuel and Boddy [1] evaluated 158 children with LUTS, including 58 cases with SBO (36\%), and they concluded that SBO was probably a coincidental finding and its true significance in children with LUTS could not be established. In addition, Kumar et al. [15] reported that there was no significant difference in the incidence of SBO between children with or without nocturnal enuresis. Notably, most studies, which generated inconsistent data regarding the relationship between radiographic SBO and urinary voiding problems, were done in children. We thus decided to perform a cross-sectional study in middle-aged and elderly people in our city using a randomized, double-blind design and we believed that our results were reliable and clinically relevant because of fewer confounding factors in our study population.

Overactive bladder (OAB) syndrome is a common LUTS afflicting millions of people worldwide. The prevalence of $\mathrm{OAB}$ is between 3\% and 43\% [16]. In 2002, the International Continence Society revised the terminology to define $\mathrm{OAB}$ as: urgency, with or without urge incontinence, usually with frequency and nocturia, which can be described as the OAB syndrome, urge syndrome, or urgency-frequency syndrome. These terms can be used after excluding infection or obvious pathology [17]. Multiple risk factors may affect the occurrence of $\mathrm{OAB}$, such as age, diabetes, UTIs, childhood nocturnal enuresis, constipation, lack of mobility, cognitive impairment, drug therapy (e.g., diuretics), BMI $>30 \mathrm{~kg} / \mathrm{m}^{2}$, and vaginal delivery [18-21]. We therefore excluded subjects with some conditions including 
those with UTIs, lack of mobility, cognitive impairment, and diuretics therapy. Our results indicated that age, SBO, HCI, and constipation were risk factors for $\mathrm{OAB}$ among general subjects, as well as age, $\mathrm{SBO}$, and constipation in men and age, $\mathrm{SBO}$, and $\mathrm{HCI}$ in women $(\mathrm{P}<0.05)$.

While age is a well-established risk factor, the role of HCE, obesity, constipation, $\mathrm{DM}$, and vaginal delivery in $\mathrm{OAB}$ remain elusive. Our study showed that constipation had a different impact on men and women for $\mathrm{OAB}$, suggesting a role of anatomical differences in the pelvic cavity. Because the rectum is adjacent to the bladder in men, constipation with rectal distension puts direct pressure on the posterior bladder wall causing bladder overactivity, trigonal irritation, post bladder wall invagination, bladder and urethral obstruction, or distension [22]. In contrast, as the bladder and rectum are separated by the vagina and uterus in women, a dilated rectum does not have a direct impact on the bladder.

A plausible explanation for the different effect of $\mathrm{HCI}$ on $\mathrm{OAB}$ in men and women might be due to the different diagnostic criteria for cerebral infarction in different hospitals (the data about HCI were obtained orally from subjects who obtained their diagnoses of cerebral infarction from different doctors and hospitals).

Somewhat surprisingly, our results indicated that $\mathrm{BPH}$ was not a risk factor for $\mathrm{OAB}$, which was not consistent with some previous observations. We reasoned that our cross-sectional study design did make a difference in findings as compared to retrospective investigations. Our screening demonstrated that many men with BPH did not have any urinary symptoms, suggesting asymptomatic BPH did not affect bladder function and thus was not a risk factor for OAB. This possibility was confirmed by the relationship between the BPH severity score and the existence of $\mathrm{OAB}$.

Little information is available regarding SBO being a potential risk factor for $\mathrm{OAB}$. Our logistic analysis of the relationship between $\mathrm{SBO}$ and $\mathrm{OAB}$ showed an OR value of 4.91 with the $95 \%$ confidence interval being 3.2-7.53. The risk of suffering $\mathrm{OAB}$ in persons with $\mathrm{SBO}$ was 5 times higher than for people without SBO, which was consistent with Fidas's observation [5]. An urodynamic study of 43 patients with SBO showed phasic detrusor overactivity in $72 \%$ cases [4]. Similarly, Guerra et al. [23] demonstrated that detrusor overactivity was present in $71 \%$ (17 of 24) of children with SBO [23]. Thus, it is reasonable to reach the conclusion that $\mathrm{SBO}$ is a real deformity, instead of a normal anatomical variant, and it plays a role in $\mathrm{OAB}$ in adults.
The precise mechanism responsible for the formation of $\mathrm{OAB}$ remains largely unknown. An important reason is that $\mathrm{OAB}$ is a diagnosis based on clinical manifestations and patients complaints, which makes it difficult to establish a reliable animal model to test different hypotheses. Three main theories have been proposed regarding the cause of $\mathrm{OAB}$ and detrusor overactivity, namely, the myogenic or muscle related theory [24], the autonomous bladder theory [25], and the neurogenic or nerve related theory [26]. SBO leading to OAB may be related to the third theory - the nerve related theory, implying that damage to central inhibitory pathways in the brain or spinal cord or sensitization of peripheral afferent terminals in the bladder can unmask primitive voiding reflexes that trigger detrusor overactivity.

Several hypotheses have been proposed to explain the role of $\mathrm{SBO}$ in $\mathrm{OAB}$. The first hypothesis is that those outcomes were secondary to a reduction in oxygen metabolism in the mitochondria of the neuronal cells of the tethered lumbosacral cord [27]. The traction on the cord between 2 fixation points leads to stretching, kinking, and distortion of the arterioles, venules, and capillaries to the spinal cord, resulting in intermittent neuronal hypoxia and accumulated damage. Increased growth has been hypothesized to cause neurological deterioration. Metabolic injury occurs preferentially to spinal neurons with higher oxygen requirements, such as interneuronal axon connections that lead to the clinical manifestation of bladder dysfunction. In contrast, the long neuronal tracts affecting muscular movements are less susceptible to early hypoxic injury, which may explain the early, often isolated appearance of bladder dysfunction and later appearance of lower limb findings in the TCS [28]. Detrusor hyperreflexia is the most common urodynamic finding, which is in contrast to other types of bladder dysfunction.

A second hypothesis speculates that $\mathrm{SBO}$ related to detrusor overactivity is associated with the emergence of a capsaicinsensitive C-fiber-mediated spinal micturition reflex caused by a reorganization of synaptic connections in the spinal cord. These C-fibers that are usually silent during a normal state of bladder filling become active during a pathological state and can fire even at low pressures [29]. During spinal cord injury, the bladder afferents, which normally are unresponsive to low intravesical pressures, become more mechanosensitive, leading to the development of detrusor overactivity. The mechanism underlying the increased mechanosensitivity of C-fibers may be plasticity of the dorsal root ganglion cells supplying the bladder as 
evidenced by enlargement of these cells and increased electrical excitability [29].

The third hypothesis suggests that dysplasia of the spinal cord may interfere with nerve conduction by a gradual process. It is postulated that the extrinsic anomalies strangulate or limit movement of nerve structures within the theca and fat deposits may cause pressure on spinal structures [14].

Although the mechanism for SBO mediated OAB is not fully understood, accumulating evidence suggests that clinical symptoms are caused by SBO combined with TCS or other spinal cord abnormalities.

A critical question remains unanswered: how can a congenital condition have its first clinical manifestation only at an adult age? It is thought that with subtle tethering, SBO will remain asymptomatic during childhood, and cumulative micro damage from repeated insults throughout life results in adult onset TCS [30]. Therefore, an otherwise asymptomatic, mild tethering may become symptomatic over the years. In our opinion, there may be no spinal cord tethering in children with SBO when they are born. However, compared with those without $\mathrm{SBO}$, they are more vulnerable to spinal cord injury during the following conditions: repeated spinal flexion, pregnancy and parturition, long-term heavy lifting, and intense activities.

We acknowledge the limitations of this study: the lack of spinal magnetic resonance imaging (MRI) and urodynamic studies because of insufficient financial support. However, we will perform these examinations in our follow-up studies, although a previous report indicates that patients with SBO and LUTS may not need to undergo spinal MRI [31].

In conclusion, the prevalence of $\mathrm{SBO}$ among the Chinese is significantly higher than that among the English, indicating that $\mathrm{SBO}$ might be region- and race-dependent. In addition, the prevalence of SBO is significantly higher among men than women, presumably reflecting genetic, ethnic, and environmental factors, which are at present poorly understood. Furthermore, SBO could gradually lead to dysfunction of spinal nerves and LUTS, such as OAB; therefore, rather than a normal variation as suggested by other researchers, $\mathrm{SBO}$ is a real deformity and a novel risk factor for $\mathrm{OAB}$.

\section{ACKNOWLEDGEMENTS}

We thank radiologists Qiang Wu, Jianbo Gao, and the members of the Center of Health Examination in the First Affiliated Hospital of Zhengzhou University for offering their important help.

\section{REFERENCES}

1. Samuel M, Boddy SA. Is spina bifida occulta associated with lower urinary tract dysfunction in children? J Urol 2004;171(6 Pt 2):26646.

2. Warder DE. Tethered cord syndrome and occult spinal dysraphism. Neurosurg Focus 2001;10:e1.

3. Nejat F, Radmanesh F, Ansari S, Tajik P, Kajbafzadeh A, El Khashab M. Spina bifida occulta: is it a predictor of underlying spinal cord abnormality in patients with lower urinary tract dysfunction? J Neurosurg Pediatr 2008;1:114-7.

4. Galloway NT, Tainsh J. Minor defects of the sacrum and neurogenic bladder dysfunction. Br J Urol 1985;57:154-5.

5. Fidas A, MacDonald HL, Elton RA, McInnes A, Wild SR, Chisholm GD. Prevalence of spina bifida occulta in patients with functional disorders of the lower urinary tract and its relation to urodynamic and neurophysiological measurements. BMJ 1989; 298:357-9.

6. Yamaguchi O, Nishizawa O, Takeda M, Yokoyama O, Homma Y, Kakizaki H, et al. Clinical guidelines for overactive bladder. Int J Urol 2009;16:126-42.

7. Fidas A, MacDonald HL, Elton RA, Wild SR, Chisholm GD, Scott R. Prevalence and patterns of spina bifida occulta in 2707 normal adults. Clin Radiol 1987;38:537-42.

8. Kumar A, Tubbs RS. Spina bifida: a diagnostic dilemma in paleopathology. Clin Anat 2011;24:19-33.

9. Sakakibara R, Hattori T, Uchiyama T, Kamura K, Yamanishi T. Uroneurological assessment of spina bifida cystica and occulta. Neurourol Urodyn 2003;22:328-34.

10. Boone D, Parsons D, Lachmann SM, Sherwood T. Spina bifida occulta: lesion or anomaly? Clin Radiol 1985;36:159-61.

11. Northrup H, Volcik KA. Spina bifida and other neural tube defects. Curr Probl Pediatr 2000;30:313-32.

12. Sutow WW, Pryde AW. Incidence of spina bifida occulta in relation to age. AMA J Dis Child 1956;91:211-7.

13. Mehdizadeh M, Roohi A, Hemami M, Esfahani ST. Is there any association between Spina bifida occulta and primary vesicoureteral reflux? Iran J Pediatr 2010;20:348-52.

14. Gregerson DM. Clinical consequences of spina bifida occulta. J Manipulative Physiol Ther 1997;20:546-50.

15. Kumar P, Aneja S, Kumar R, Taluja V. Spina bifida occulta in functional enuresis. Indian J Pediatr 2005;72:223-5.

16. Hashim H, Abrams P. Overactive bladder: an update. Curr Opin Urol 2007;17:231-6.

17. Abrams P, Cardozo L, Fall M, Griffiths D, Rosier P, Ulmsten U, et 
al. The standardisation of terminology of lower urinary tract function: report from the Standardisation Sub-committee of the International Continence Society. Neurourol Urodyn 2002;21:167-78.

18. Wang Y, Xu K, Hu H, Zhang X, Wang X, Na Y, et al. Prevalence, risk factors, and impact on health related quality of life of overactive bladder in China. Neurourol Urodyn 2011;30:1448-55.

19. Fitzgerald MP, Thom DH, Wassel-Fyr C, Subak L, Brubaker L, Van Den Eeden SK, et al. Childhood urinary symptoms predict adult overactive bladder symptoms. J Urol 2006;175(3 Pt 1):989-93.

20. Zhang W, Song Y, He X, Huang H, Xu B, Song J. Prevalence and risk factors of overactive bladder syndrome in Fuzhou Chinese women. Neurourol Urodyn 2006;25:717-21.

21. Freeman RM, Adekanmi OA. Overactive bladder. Best Pract Res Clin Obstet Gynaecol 2005;19:829-41.

22. Chase J, Austin P, Hoebeke P, McKenna P; International Children's Continence Society. The management of dysfunctional voiding in children: a report from the Standardisation Committee of the International Children's Continence Society. J Urol 2010;183:1296302.

23. Guerra LA, Pike J, Milks J, Barrowman N, Leonard M. Outcome in patients who underwent tethered cord release for occult spinal dys- raphism. J Urol 2006;176(4 Pt 2):1729-32.

24. Brading AF. A myogenic basis for the overactive bladder. Urology 1997;50(6A Suppl):57-67.

25. Drake MJ, Mills IW, Gillespie JI. Model of peripheral autonomous modules and a myovesical plexus in normal and overactive bladder function. Lancet 2001;358:401-3.

26. de Groat WC. A neurologic basis for the overactive bladder. Urology 1997;50(6A Suppl):36-52.

27. Fujita Y, Yamamoto H. An experimental study on spinal cord traction effect. Spine (Phila Pa 1976) 1989;14:698-705.

28. Giddens JL, Radomski SB, Hirshberg ED, Hassouna M, Fehlings M. Urodynamic findings in adults with the tethered cord syndrome. J Urol 1999;161:1249-54.

29. Banakhar MA, Al-Shaiji TF, Hassouna MM. Pathophysiology of overactive bladder. Int Urogynecol J 2012;23:975-82.

30. Veenboer PW, Bosch JL, van Asbeck FW, de Kort LM. Paucity of evidence for urinary tract outcomes in closed spinal dysraphism: a systematic review. BJU Int 2013;112:1009-17.

31. Pippi Salle JL, Capolicchio G, Houle AM, Vernet O, Jednak R, O'Gorman AM, et al. Magnetic resonance imaging in children with voiding dysfunction: is it indicated? J Urol 1998;160(3 Pt 2):1080-3. 